\title{
Robust Cooperative Close Formation Flight Control of Multiple Unmanned Aerial Vehicles
}

\author{
Qingrui Zhang \\ The Institute for Aerospace Studies University of Toronto \\ (UTIAS) \\ 4925 Dufferin St. Toronto, Canada M3H 5T6 \\ qingrui.zhang@mail.utoronto.ca
}

\begin{abstract}
This paper investigates the close formation control problem of multiple unmanned aerial vehicles (UAVs). A robust cooperative control algorithm is proposed in light of the uncertainty and disturbance estimation technique. In the proposed design, each UAV is assigned a virtual leader which defines the desired position for the corresponding UAV in close formation. Bidirectional communication topology is assumed for UAVs in close formation, based on which a cooperative control law is thereafter established on each UAV. Model uncertainties and formation aerodynamic disturbances are efficiently estimated and compensated using a uncertainty and disturbance estimator. Eventually, the efficacy of the proposed design will be validated via the close formation simulation of five aircraft.
\end{abstract}

Keywords-component; close formation control; cooperative control; Multi-UAVs; uncertainty and disturbacne estimation

\section{INTRODUCTION}

Close formation flight stems from the formation flight of migrating birds [1]. It has received extensive research attention in past decades [2, 3]. The research interest in close formation flight is motivated by its potential in drag reductions and fuel savings. When an aircraft is flying in air, trailing vorticescircular patterns of rotating airs-will be left behind it. The trailing vortices are divided into downwash wake and upwash wake. If a follower aircraft is managed to fly at certain proper relative position to a leader aircraft, the upwash wake could be used to reduce the drag of the follower aircraft, thereby decreasing energy expenditure. According to flight tests by NASA, close formation flight can generate 13\%-20\% drag reductions and $14 \%-18 \%$ fuel savings on a follower aircraft [4]. Wind-tunnel experimental studies by Cho et al indicate $12 \%$ increase in the lift-to-drag ratio of a follower aircraft in close formation against solo flight [5].

However, close formation flight is always a challenging problem for unmanned aerial vehicles. Highly efficient and accurate formation control algorithms are indispensable, as close formation flight only makes sense when a follower is keeping a certain optimal position to its leader. The theoretical

\author{
Hugh H. T. Liu \\ The Institute for Aerospace Studies University of Toronto \\ (UTIAS) \\ 4925 Dufferin St. Toronto, Canada M3H 5T6 \\ liu@uitas.utoronto.ca
}

analysis by the authors shows more 30\% drag reduction by close formation flight could be lost, if a follower fails to track the optimal relative position by $10 \%$ wing span [6]. In addition, the trailing vortices, working like wind gusts, will induce other adverse aerodynamic disturbances on the follower aircraft, which increases the difficulty of the control design [6].

So far, the close formation control problem has been studied using different methods, such as PI controller [7], sliding mode control [8], LQR control [9], MPC control [10], adaptive control [11], and robust control [12]. However, all of them were developed under the leader-follower architecture, and there was no cooperation between two UAVs. The efficacy of the existing methods can only be guaranteed for close formation flight of two or three aircraft. Increase of formation size (number of UAVs) will result in dramatic loss of efficiency and accuracy for the existing methods. To deal with the deficiency of the existing methods in close formation flight of more than three UAVs, a cooperative control method is proposed in this paper. A bidirectional communication topology is employed. UAVs in close formation are required to communicate with some of their neighbors. To enhance the robustness against model uncertainties and formation aerodynamic disturbances, the uncertainty and disturbance estimation technique is employed and combined with the proposed cooperative formation controller. The efficiency of the proposed control algorithm is verified via the close formation simulation of five aircraft.

The paper is organized as follows. In section II, a nonlinear UAV model is introduced together with the close formation aerodynamics. Section III presents the robust cooperative control methods. The numerical simulations are given in Section IV. Eventually, some conclusion remarks are provided in Section V.

\section{PRELIMINARIES}

\section{A. Aircraft model}

The proposed cooperative control law will perform as an outer-loop formation tracking controller. Hence, a 6-state nonlinear UAV model is presented for the control design. 


$$
\left\{\begin{array}{l}
\dot{x}_{i}=v_{i} \cos \chi_{i}, \dot{y}_{i}=v_{i} \sin \chi_{i}, \dot{h}_{i}=\eta_{i} \\
\dot{v}_{i}=\left(T_{i}-D_{i}-\Delta D_{i}\right) / m_{i} \\
\dot{\chi}_{i}=\left(L_{i}+\Delta L_{i}\right) \sin \mu_{i} /\left(m_{i} v_{i}\right) \\
\dot{\eta}_{i}=\left(L_{i}+\Delta L_{i}\right) \cos \mu_{i} / m_{i}-g
\end{array}\right.
$$

where $x_{i}, y_{i}$, and $h_{i}$ denote the position of the i-th UAV in the inertial frame, $v_{i}$ is the ground speed projection on a horizontal plane, $\eta_{i}$ is the climbing rate, $\chi_{i}$ is the heading angle, $\mu_{i}$ is the bank angle, $L_{i}$ is the lift, $D_{i}$ is the drag, $T_{i}$ is the thrust, $g$ is the gravity acceleration, $\Delta D_{i}$ and $\Delta L_{i}$ aerodynamic forces induced by the trailing vortices of leader aircraft. The control inputs are chosen to be $T_{i}, \mu_{i}$, and $L_{i}$. The vortex-induced forces $\Delta D_{i}$ and $\Delta L_{i}$ are given by

$$
\begin{aligned}
& \Delta L_{i}=\int_{-\frac{b}{2}}^{\frac{b}{2}} q_{\infty} c(s) C_{l_{\alpha}} \Delta \alpha(s) d s \\
& \Delta D_{i}=L_{i}^{\prime} \sin (\overline{\Delta \alpha})=\left(L_{i}+\Delta L_{i}\right) \sin (\overline{\Delta \alpha})
\end{aligned}
$$

where $\Delta \alpha$ is the vortex-induced change of the angle of attack along the wing span as shown in Figure 1, while $\overline{\Delta \alpha}$ is the effective average change of the angle of attack. Notice that $\Delta L_{i}$ and $\Delta D_{i}$ are actually functions of the relative positions between a follower aircraft and its leader. Shown in Figure 2 and 3 are the relationships between non-dimensional lift and drag coefficients with respect to the lateral and vertical separations. More detailed results can be found at [6].

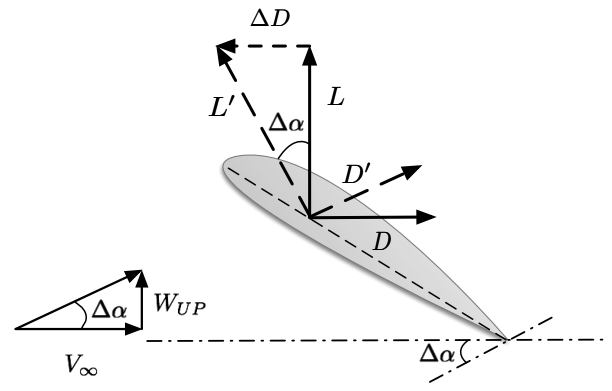

Figure 1: Lift rotation due to the upwash wake

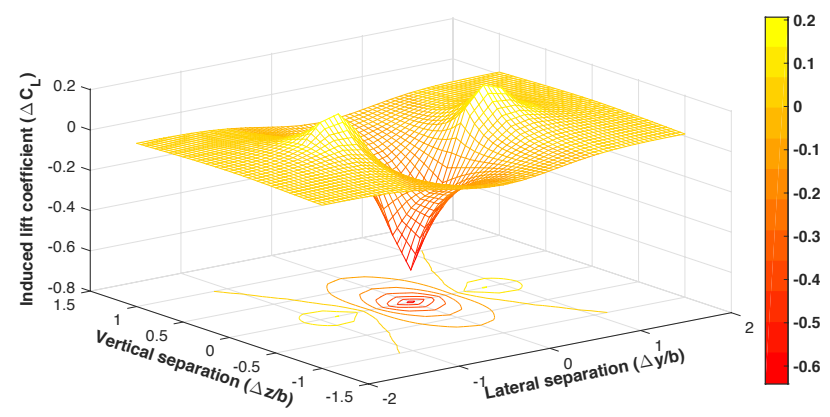

Figure 2: Vortex-induced lift coefficient variation

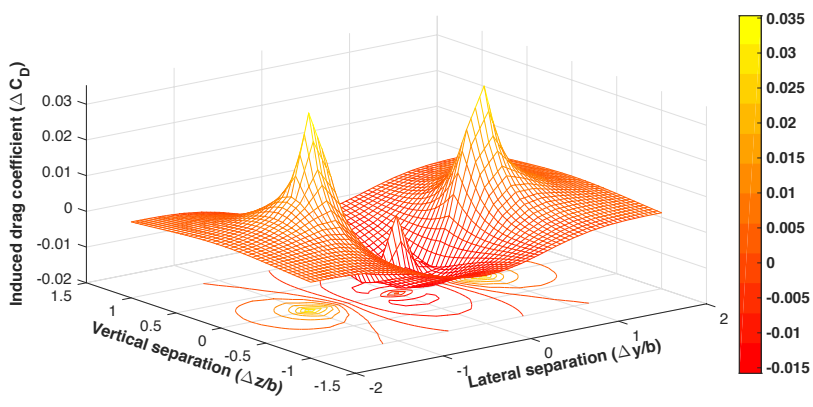

Figure 3: Vortex-induced drag coefficient variation

\section{B. Control conversion}

In the control design, $D_{i}$ is taken as part of the model uncertainties. Differentiating the kinematic equations of Model (1) will yield

$$
\left\{\begin{array}{c}
\ddot{x}_{i}=\dot{v}_{i} \cos \chi_{i}-v_{i} \dot{\chi}_{i} \sin \chi_{i} \\
\ddot{y}_{i}=\dot{v}_{i} \sin \chi_{i}+v_{i} \dot{\chi}_{i} \cos \chi_{i} \\
\ddot{h}_{i}=\dot{\eta}_{i}
\end{array}\right.
$$

Define

$u_{x i}=\frac{T_{i}}{m_{i}} \cos \chi_{i}-\frac{L_{i}}{m_{i}} \sin \mu_{i} \sin \chi_{i}$

$u_{y i}=\frac{T_{i}}{m_{i}} \sin \chi_{i}+\frac{L_{i}}{m_{i}} \sin \mu_{i} \cos \chi_{i}$

$u_{z i}=\frac{L_{i}}{m_{i}} \cos \mu_{i}-g$

$d_{x i}=-\frac{D_{i}+\Delta D_{i}}{m_{i}} \cos \chi_{i}-\frac{\Delta L_{i}}{m_{i}} \sin \mu_{i} \sin \chi_{i}$

$d_{y i}=-\frac{D_{i}+\Delta D_{i}}{m_{i}} \sin \chi_{i}+\frac{\Delta L_{i}}{m_{i}} \sin \mu_{i} \cos \chi_{i}$

$d_{z i}=\frac{\Delta L_{i}}{m_{i}} \cos \mu_{i}$

Eventually, the nonlinear model is transformed into a standard $2^{\text {nd }}$-oder integrator model as shown in (2).

$$
\ddot{\rho}_{i}=u_{\rho i}+d_{\rho i} \quad\left(\rho \in\left\{\begin{array}{lll}
x & y & z
\end{array}\right\}\right)
$$

\section{ROBUST COOPERATIVE CONTROL}

The entire control law is

$$
u_{\rho i}=u_{\rho 0 i}-\hat{d}_{\rho i} \quad\left(\rho \in\left\{\begin{array}{lll}
x & y & z
\end{array}\right\}\right)
$$


where $u_{\rho 0 i}$ is the baseline cooperative control, $\hat{d}_{\rho i}$ is the estimation of the uncertainties and disturbances.

\section{A. Baseline cooperative control}

In this subsection, a cooperative baseline control law is proposed for close formation flight of $\mathrm{N}$ UAVs where $\mathrm{N}>2$. The major expectation is to increase the scalability, efficiency, and robustness of close formation flight by introducing certain cooperative mechanism. In the cooperative control method, a virtual leader together is introduced to generate the reference signals for each vehicle. All the virtual leaders together defines the optimal formation shape (usually, "V-shape") for a group of N UAVs, as shown in Figure 4. A bidirectional communication topology is employed.

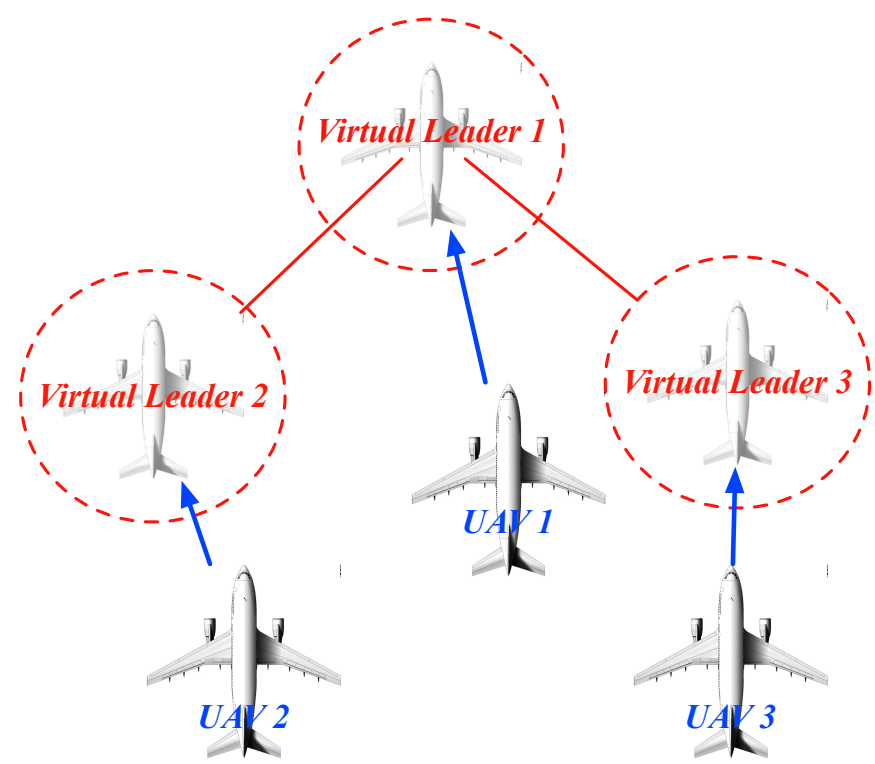

Figure 4 : Close formation organization

Define the position of each virtual leader as $\vec{r}_{i}=\left[\begin{array}{lll}r_{x i} & r_{y i} & r_{z i}\end{array}\right]^{T}$. Assume the $1^{\text {st }}$ and $2^{\text {nd }}$ of $r_{x i}, r_{y i}$, and $r_{z i}$ are available. Define the position tracking error as $e_{\rho i}=\rho_{i}-r_{\rho i}$. The baseline cooperative control law for i-th $\mathrm{UAV}$ is

$$
\begin{array}{rc}
u_{\rho 0 i}= & \ddot{r}_{\rho i}-K_{P \rho} e_{\rho i}-K_{V \rho} \dot{e}_{\rho i} \\
& -\sum_{j \in \mathbb{N}_{i}}\left[C_{P \rho}\left(e_{\rho i}-e_{\rho j}\right)+C_{V \rho}\left(\dot{e}_{\rho i}-\dot{e}_{\rho j}\right)\right]
\end{array}
$$

where $\rho \in\left\{\begin{array}{lll}x & y & z\end{array}\right\}, \quad K_{P \rho}, K_{V \rho}, C_{P \rho}, C_{V \rho}>0$, $\mathbb{N}_{i}$ denotes the set of neighbors of $i$-th aircraft.

\section{B. Uncertainty and disturbance estimator}

The uncertainty and disturbance estimation term $\hat{d}_{\rho i}$ is designed by applying (4) to (3).

$$
\ddot{\rho}_{i}=u_{\rho 0 i}-\hat{d}_{\rho i}+d_{\rho i} \quad\left(\rho \in\left\{\begin{array}{lll}
x & y & z
\end{array}\right\}\right)
$$

In terms of results in [], $d_{\rho i}$ is estimated using a stable lowpass first-order filter $G_{\rho i}(s)$, where

$$
G_{\rho i}(s)=\frac{1}{T_{\rho i} s+1}
$$

Hence, $\hat{d}_{\rho i}$ is given by

$$
\hat{d}_{\rho i}=\frac{1}{T_{\rho} s+1} d_{\rho i}
$$

where $T_{\rho}$ is the time constant, $d_{\rho i}$ is usually unavailable. However, $d_{\rho i}$ is equal to $\ddot{\rho}_{i}-u_{\rho 0 i}+\hat{d}_{\rho i}$ according to Eq. (5). Replace $d_{\rho i}$ by $\ddot{\rho}_{i}-u_{\rho 0 i}+\hat{d}_{\rho i}$ in (6). Via basic mathematical derivations, we can get

$$
\hat{d}_{\rho i}=\frac{1}{T_{\rho} S}\left(\ddot{\rho}_{i}-u_{\rho 0 i}\right)
$$

Specifying (7) in the time-domain will yield

$$
\hat{d}_{\rho i}=\frac{1}{T_{\rho}}\left(\dot{\rho}_{i}(t)-\dot{\rho}_{i}(0)-\int_{0}^{t} u_{\rho 0 i} d \tau\right)
$$

where $\dot{\rho}_{i}(t)$ is a measurable speed signal which makes (8) always practically applicable.

\section{NumericAl Simulations}

In the numerical simulations, close formation flight of 5 UAVs is considered. Among the $5 \mathrm{UAVs}$, one UAV performs as the very leader for the entire formation. This formation leader will be in charge of tracking the trajectory defined for the entire formation. The virtual leaders are defined according to the optimal relative position requirements to the formation leader. In the simulation results, the leader UAV for the entire formation is labelled to be "Leader", while the other UAVs are labelled to be "Follower 1", "Follower 2", "Follower 3", and "Follower 4", respectively. The control gains are

$$
\begin{array}{ll}
K_{P x}=K_{P y}=K_{P z}=1.5, & K_{V x}=K_{V y}=K_{V z}=3 \\
C_{P x}=C_{P y}=C_{P z}=1, & C_{V x}=C_{V y}=C_{V z}=2
\end{array}
$$




$$
T_{x}=T_{y}=T_{z}=0.1
$$

The communication topology of the five UAVs are shown in Figure 5. The formation trajectory tracking performance is shown in Figure 6 in which the 3-D trajectory is projected on a horizontal plane. Detailed tracking error responses are shown in Figure 7.

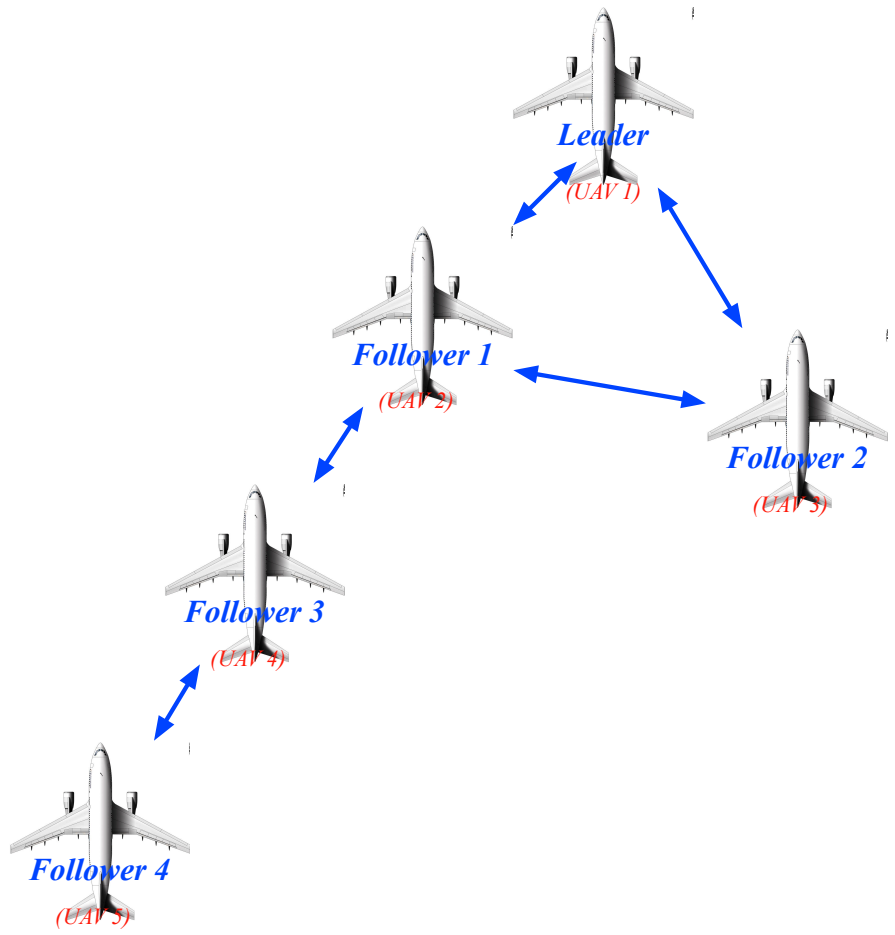

Figure 5 : Communication Topology

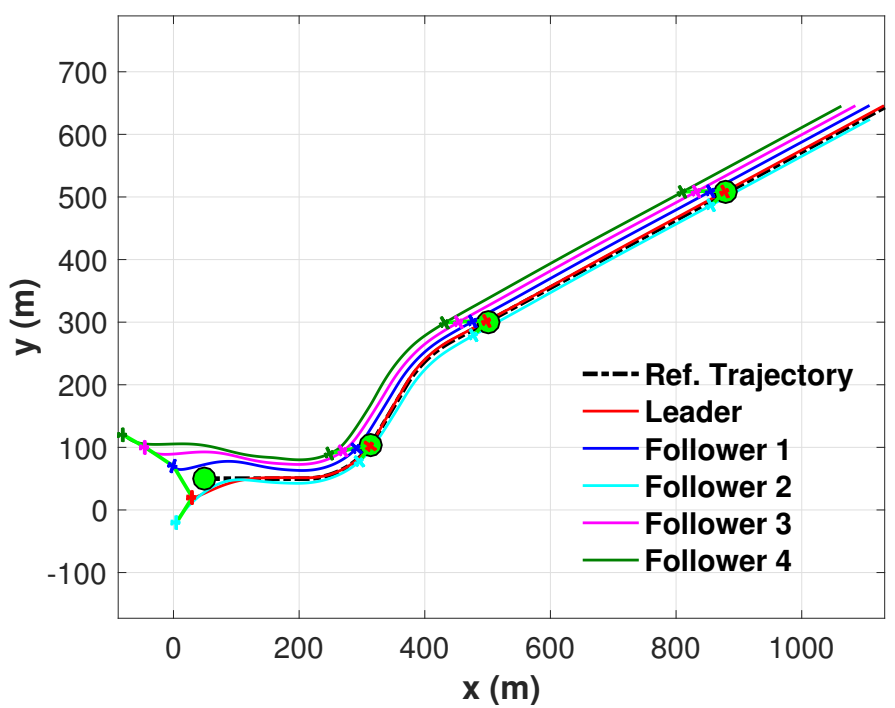

Figure 6: Formation trajectory projection on a 2-D horizontal plane
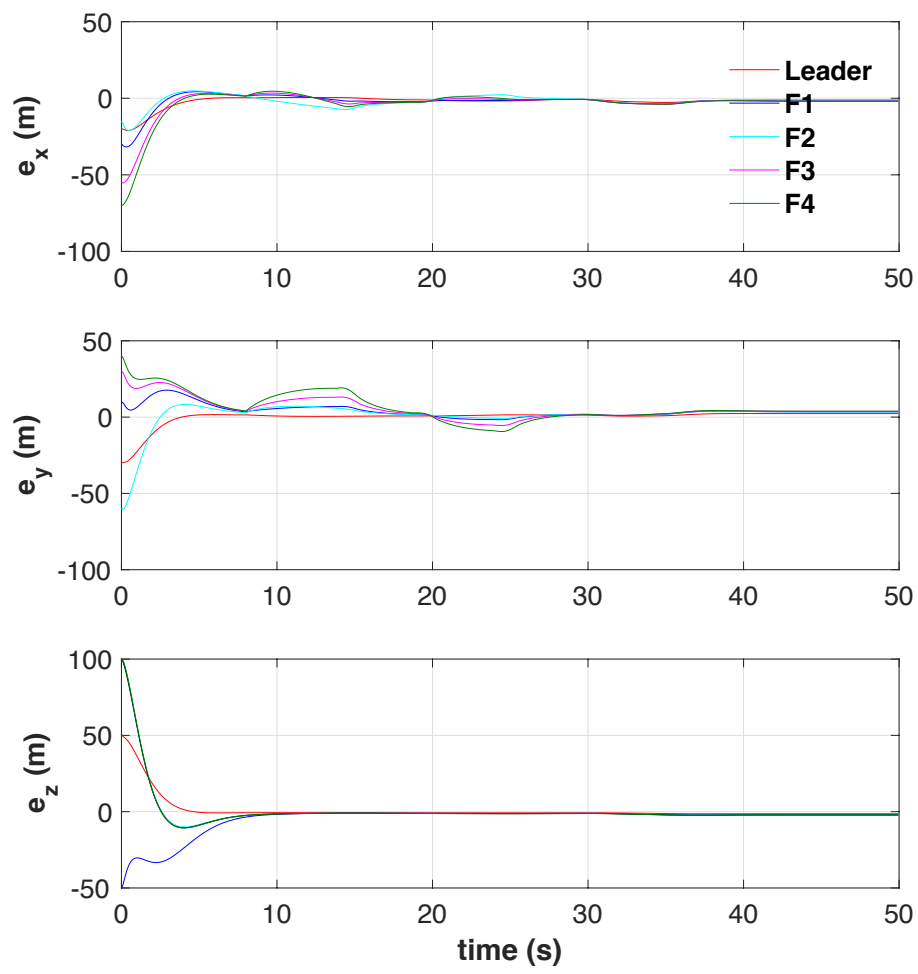

Figure 7: Formation tracking error responses

\section{CONCLUSION}

This paper presents an efficient robust cooperative close formation control algorithms. Based on the proposed design, close formation flight can be easily extended to the case of more than three UAVs. The efficacy of the proposed design is verified via a close formation simulation of five UAVs.

\section{REFERENCES}

[1] Portugal, S. J., Hubel, T. Y., Fritz, J., and et al, "Upwash exploitation and downwash avoidance by ap phasing in ibis formation flight," Nature, Vol. 413, pp. 399-402, 2014.

[2] Fowler, J. M. and D'Andrea, R., "Distributed control of close formation flight," Proceedings of the 41st IEEE Conference on Decision and Control, IEEE, Las Vegas, Nevada USA, 2002.

[3] Brodecki, M. and Subbarao, K., "Autonomous Formation Flight Control System Using In-Flight Sweet-Spot Estimation,” Journal of Guidance, Control, and Dynamics, Vol. 38, No. 6, pp. 1083-1096, 2015.

[4] Ray, R., Cobleigh, B., Vachon, M. J., and John, C. S., "Flight Test Techniques Used to Evaluate Performance Benefits During Formation Flight," Proceedings of AIAA Atmospheric Flight Mechanics Conference and Exhibit, AIAA, Monterey, California, AIAA 2002-4492, 2002.

[5] Cho, H., Lee, S., and Han, C., "Experimental study on the aerodynamic characteristics of a ghter-type aircraft model in close formation ight," Journal of Mechanical Science and Technology, Vol. 28, No. 8, pp. 3059-3065, 2014.

[6] Q. Zhang and H. H. T. Liu, "Aerodynamics modeling and analysis of close formation flight," Journal of Aircraft, vol. 54, no. 6, pp. 2192 2204, 2017.

[7] M. Pachter, J. J. D. Azzo, and A. W. Proud, "Tight formation flightcontrol," Journal of Guidance, Control, and Dynamics, vol. 24, no. 2, pp. 246-254, 2001.

[8] S. Singh, M. Pachter, P. Chandler, S. Banda, S. Rasmussen, and C. Schumacher, "Input-output invertibility and sliding mode control for 
close formation flying," Int. J. Robust Nonlinear Control, vol. 10, no. 10, pp. 779-797, 2000.

[9] A. Dogan and S. Venkataramanan, "Nonlinear control for reconfiguration of unmanned-aerial-vehicle formation," Journal of Guidance, Control, and Dynamics, vol. 28, no. 4, pp. 667-678, 2004.

[10] F. A. de Almeida, "Tight formation flight with feasible model predictive control," in Proceedings of AIAA Guidance, Navigation, and Control Conference. Kissimmee, Florida, U.S.A.: AIAA, 2015, AIAA 20150602.

[11] Q. Zhang and H. H.-T. Liu, "Integrator-augmented robust adaptive control design for close formation flight," in Proceedings of 2017 AIAA
Guidance, Navigation, and Control Conference. Grapevine, Texas: AIAA, 2017.

[12] Q. Zhang and H. H.-T. Liu, "Robust design of close formation flight control via uncertainty and disturbance estimator," in Proceedings of 2016 AIAA Guidance, Navigation, and Control Conference. San Diego, California, USA: AIAA, 2016, AIAA 2016-2102.

[13] Q. Zhang and H. H. T. Liu, "UDE-based robust command filtered backstepping control for close formation flight," IEEE Transactions on Industrial Electronics, 10.1109/TIE.2018.2811367. 